(C) Inderscience Enterprises Ltd. The original source of publication is available at InderScience. Petersen, W., \& McPhee, J. (2014). A study of volumetric contact modelling approaches in rigid tyre simulation for planetary rover application. International Journal of Vehicle Design, 64(2-4), 262-279. https://doi.org/10.1504/IJVD.2014.058489

\title{
A Study of Volumetric Contact Modelling Approaches in Rigid Tire Simulation for Planetary Rover Application
}

\author{
W. Petersen* \\ Department of Systems Design Engineering, \\ University of Waterloo, \\ Waterloo, Ontario, Canada \\ E-mail: wpeterse@engmail.uwaterloo.ca \\ ${ }^{*}$ Corresponding author
}

\section{J. McPhee}

Department of Systems Design Engineering, University of Waterloo, Waterloo, Ontario, Canada

E-mail: mcphee@real.uwaterloo.ca

\begin{abstract}
For planetary rover applications, a volumetric contact modelling approach is used to capture the dynamics of the rigid tire/soil interface. The volumetric contact model allows for determining closed-form expressions for the tire contact forces. These volumetric force representations contain information about the shape of the contact geometry so that the analytical expressions result in fast simulations. Three different volumetric rigid tire models are developed and evaluated from a plasticity point of view. The performance of each tire is tested and compared with respect to the resistance force caused by the ongoing compaction of the soil and the resultant plastic deformation. The quantity used to model the plastic deformation of the soil is represented by the soil rebound. Moreover, each tire model is compared against experimental data to evaluate its validity.
\end{abstract}

Keywords: contact mechanics; volumetric contact model; tire/soil interaction; planetary rover simulation; analytical contact model

Reference to this paper should be made as follows: Authors (2011) 'A Study of Volumetric Contact Modelling Approaches in Rigid Tire Simulation for Planetary Rover Application', Int. J. Vehicle Design, Vol. 1, Nos. 1/1, pp.1-??.

Biographical notes: Willem Petersen did his Diplomingenieur in theoretical Mechanical Engineering from the Hamburg Institute of Technology. Willem is currently doing his $\mathrm{PhD}$ in Systems Design Engineering at the University of Waterloo, Ontario, Canada. He is working in the field of contact mechanics on problems related to offroad tire modelling for planetary rover simulations.

Copyright (c) 2009 Inderscience Enterprises Ltd. 
John McPhee received his $\mathrm{PhD}$ in Mechanical Engineering from the University of Waterloo, Canada, in 1990. He worked at the Université de Liège, Belgium, and Queen's University, Canada, before taking a faculty position in 1992 at Waterloo, where he is now a Professor in Systems Design Engineering, and the NSERC/Toyota/Maplesoft Industrial Research Chair in Mathematics-based Modelling and Design. Dr. McPhee's main area of research is multibody system dynamics, with principal application to the analysis and design of vehicles, mechatronic devices, and biomechanical systems. He has written over 150 papers, consulted to many industries in Canada and the United States, and supervised 40 graduate students and postdoctoral fellows. Dr. McPhee is an Associate Editor for seven international journals and, in 2005, he was elected a Fellow of the American Society of Mechanical Engineers. Dr. McPhee completed his term in 2009 as the Executive Director of the Waterloo Centre for Automotive Research, spending a sabbatical year at the Toyota Technical Center in Ann Arbor, Michigan.

\section{Introduction}

Rigid wheels have been used for multiple planetary and lunar exploration rover missions in the past due to their robustness and reliability. The most famous missions include the Spirit and Opportunity rovers that were sent to Mars, in the most successful rover exploration missions as of today. For the simulation of these type of planetary rovers, a crucial component of the vehicle dynamics model is the wheel/soil interaction which requires accurate prediction of the contact forces within a reasonable computation time. For these rigid tires, the major challenge is to calculate the contact forces due to the large deformations in the soil. For that purpose, three different volumetric rigid rover wheel models are developed in this paper and compared not only against each other but also against the experimental data retrieved from a single wheel testbed conducted by the German Aerospace Center (DLR) in Bremen. The volumetric contact model is used to replace the time-consuming integration of normal pressure distribution in the contact patch to determine the normal tire force. The tangential tire force on the other hand is determined in a conventional way by integrating the shear stress distribution in the contact patch, which may be described using the relationship between the shear stress and shear deformation (Wong, 2010).

The developed tire model determines the contact forces based on volumetric properties of the interpenetration volume of the contact problem (Gonthier et al., 2003). Assuming a cylindrical tire geometry on a flat terrain, it is possible to obtain a closed form solution of the volume metrics, which are the volume, center of mass and the inertia of the penetration volume. This contact modelling approach is based on a Winkler foundation model (Johnson, 1987). The volumetric contact model was developed by Gonthier et al. (2003) and it has proven to be accurate in simulation of numerous tasks in robotics. Furthermore, a validation of the volumetric normal force model was performed by Boos and McPhee (2010). The 
volumetric rigid rover wheel model is a physics-based model that is conceptually simple, which allows for an easy identification of the parameters.

Most analytical off-road tire models are based on Bekker's pressure-sinkage relation (Bekker, 1962). He used this soil representation to predict the normal pressure distribution under the tire during a rigid wheel/soil interaction (Bekker, 1969). Janosi and Hanamoto (1961) first developed a longitudinal tire force model by deriving a shear stress distribution based on Bekker's soil model and the Coulomb-Mohr failure criteria. Wong (1967) further studied the soil behaviour under a rigid tire and Onafeko and Reece (1967) determined the soil stresses based on the soil deformation caused by the undeformable geometry of a wheel. From a tire dynamics point of view, the rigid tire/soil interaction was studied in the segmented work by Wong and Reece (1967) in which the wheel performance of the driven wheel was compared against the performance of a towed wheel. Within this work the authors also discussed slip lines and the point of maximum radial pressure in the contact patch. The movement of soil particles under the impact of a driven wheel was also studied by Yong and Fattah (1975). Due to the plastic deformation in the soil caused by the wheel rolling over the soft soil, Wong (2003) proposed to separate the contact patch into sections depending on whether the soil is under compression or in relaxation. Besides the work on analytical rigid tire models, a number of deformable tire theories have been proposed. Harnisch et al. (2005) developed a tire model for a simulation tool for heavy duty off-road vehicles, whereas the tire model developed by Scharringhausen et al. (2009) was designed for application in planetary rover simulations. With a focus on planetary exploration rover projects, the rigid tire models regained popularity. Models were developed and validated using single wheel tests by Bauer et al. (2005) as well as Scharringhausen et al. (2009). A summary of these methods can be found in Wong's text books (Wong, 2003, 2010) in which he applied terramechanics theories to engineering problems in off-road vehicle design.

In this paper, three different rigid rover wheel models are developed in which the volumetric contact model is used to find a closed form solution for the normal contact forces. This modelling approach is proposed to replace the often timeconsuming integration of the normal pressure distribution given by the Bekker soil model. Moreover, the developed rigid tire models are used to evaluate the influence of the plastic deformation in the soil on the tire performance. The performance of the tire models is evaluated by determining the compaction resistance force, the generated traction force and the overall longitudinal tire force. The difference between the three volumetric tire model approaches is the geometry of the penetration volume and its dependency on the soil rebound. The soil rebound is used to model the plastic deformation by which the soil is compressed after the tire rolls over and loses contact with the terrain. The three different penetration volumes are chosen according to Wong's theory in which the material properties of a soil under compression differ from those of soils that are in relaxation. It should be noted that this paper presents the preliminary results in the development of a 3 -dimensional volumetric tire model for planetary rover simulations.

To evaluate the different rigid tire models, each model is simulated for different amounts of soil rebound and analyzed for different longitudinal tire slips at a constant wheel load. The resultant longitudinal forces are confirmed by the experimentally gained data sets. Also, it is shown that the soil rebound can be 
used to model the resistance due to plastic deformation caused by the ongoing compaction of the soil. Furthermore, the results for the soil compaction resistance and the traction force are evaluated with respect to the amount of soil rebound. The results are compared against each other and explained by means of direct and indirect relation of the soil rebound to the compaction resistance and the tractive force, respectively.

\section{Volumetric Contact Model}

Unlike most other compliant contact models used in tire modelling, which calculate the normal contact force based on the penetration depth, the tire model proposed in this paper determines contact forces based on volumetric properties of the two bodies in contact. This contact model has proven to be valid for various examples of modelling contacts in robotic task simulations (Gonthier, 2007). It uses the kinematic properties of the contact problem to find the volumetric properties of the interpenetration volume. These properties include the volume itself $V$, its centroid $\mathbf{r}_{c}$ and its inertia tensor $\mathbf{J}_{S}$ (see Figure 1).

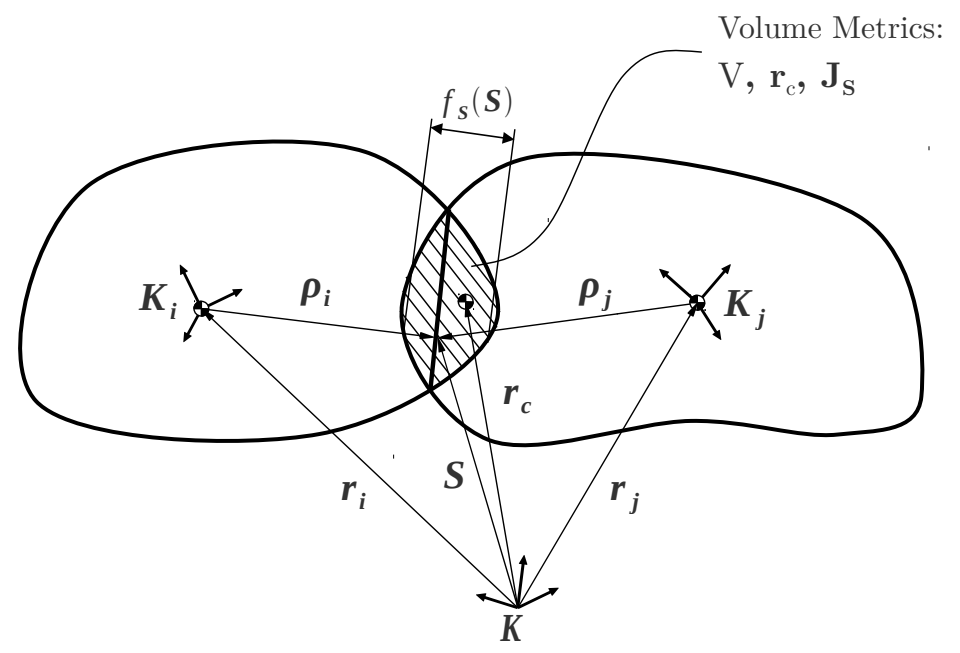

Figure 1 Schematic of volumetric contact model

The volume metrics are calculated either analytically or numerically depending on the geometries of the objects that are in contact. For simple geometries, closed form expressions can be found for Eqns $1-3$.

$$
\begin{aligned}
V & =\int_{S} f_{S}(\mathbf{S}) d S \\
\mathbf{r}_{c} & =\frac{1}{V} \int_{V} \mathbf{r} d V \\
\mathbf{J}_{S} & =\int_{S}\left[\left(\boldsymbol{\rho}_{S} \cdot \boldsymbol{\rho}_{S}\right) \mathbf{I}-\boldsymbol{\rho}_{S} \boldsymbol{\rho}_{S}\right] f_{S}(\mathbf{S}) d S
\end{aligned}
$$


where $\mathbf{S}$ describes the contact surface and $f_{S}$ is the depth of deformation at a point on the contact surface $\mathbf{S}$. The vector $\boldsymbol{\rho}_{S}$ is the position of this point relative to the location of the volume centroid and $\mathbf{I}$ is the unit tensor. These volumetric properties along with the material properties and the kinematic states of the two colliding bodies can be used to determine the contact force as explained in the following sections.

\subsection{Linear Elastic Foundation Model}

The contact model based on volumetric properties is derived for the linear elastic foundation model. The elastic foundation can be imagined as a mattress of linear elastic springs. When a body comes in contact with this foundation, the contact pressure is assumed to be proportional to the depth of deformation of the surface of the body, see Figure 2, where $k_{f}$ is the elastic modulus of the foundation and $h_{f}$ is the arbitrarily chosen depth of the foundation mattress.

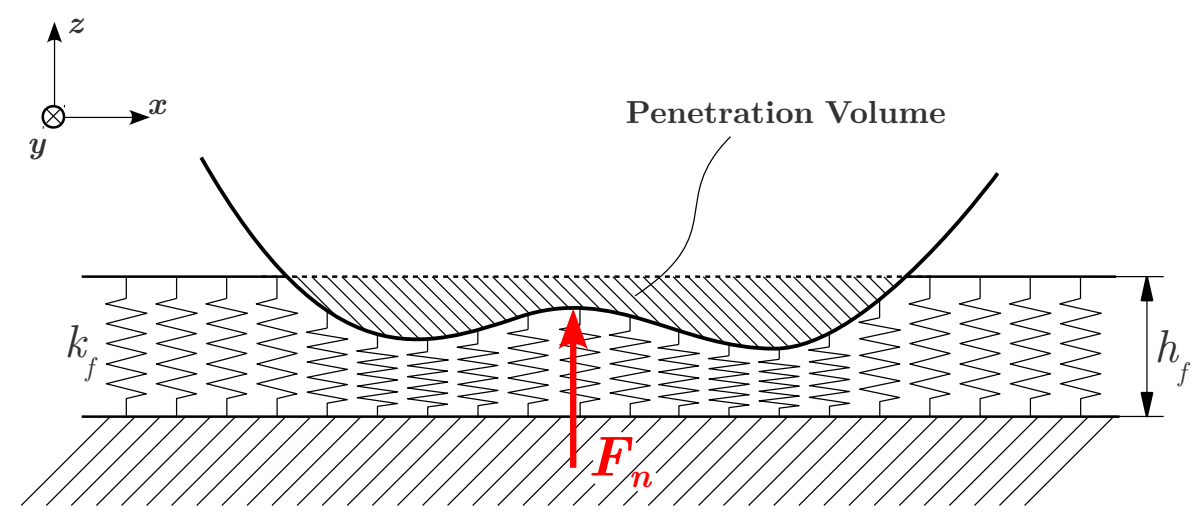

Figure 2 Schematic of Winkler foundation

By integrating the normal stress distribution of the elastic foundation over the contact surface, a general form of the normal force $\mathbf{F}_{n}$ may be described as a function of the penetration volume.

$$
\mathbf{F}_{n}=k_{v} V \mathbf{n} \text { with } k_{V}=\frac{k_{f}}{h_{f}}
$$

where $k_{V}$ is the volumetric stiffness and $\mathbf{n}$ is the vector defining the contact normal. One of the advantages of this approach is that it automatically leads to the consistent selection of the point of action of the force, which is the centroid of the volume, and the calculation of the rolling resistance moment $\boldsymbol{\tau}_{R}$ (Gonthier et al., 2003).

$$
\boldsymbol{\tau}_{R}=k_{v} d \mathbf{J}_{S} \cdot \boldsymbol{\omega}
$$

where $d$ is the damping factor and $\boldsymbol{\omega}$ is the relative angular velocity vector between the two colliding bodies. This contact model can be used for implementations 
with complex tire geometries and 3-dimensional terrains. In fact, the volumetric contact model has proven to handle multiple contacts and contacts with sharp edges (Gonthier, 2007), which have been problematic for off-road tire models in the past.

In this paper, damping in the soil is neglected. Therefore, the rolling resistance becomes zero and no inertia tensor calculation is required. In the following, the required volumetric properties are the penetration volume and its centroid.

\subsection{Hyperlastic Foundation Model}

Since most soils often react to compression in a non-linear manner, a hyperelastic foundation may be required in which the linear springs are replaced by non-linear springs. The resulting contact normal forces can then be calculated using Eqn 6 .

$$
\mathbf{F}_{n}=k_{v} \int f_{S}^{\eta}(\mathbf{S}) d S \mathbf{n}
$$

where $\eta$ is the non-linearity exponent of the hyperelastic springs. In this case, the integral of the spring displacements over the contact patch no longer equals the penetration volume, but can be described as a hypervolume. The calculation of this hypervolume to generate a non-linear volumetric contact model is part of our current research effort. Therefore, the results presented in this article are based on a linear soil possessing a Bekker exponent of $m=1$.

\section{Rigid Tire-Soil Interaction Model}

To determine the contact force of a rigid tire rolling on a compliant terrain, the soft soil must be represented by a deformable material model that is in contact with the undeformable shape of the wheel. The geometry of said wheel defines the shape of the soil compaction during impact and the level of this deformation depends on the soil properties as well as the wheel kinematics. Figure 3 shows a schematic of the described contact problem which is commonly used to model a rover wheel on soft planetary surfaces.

\subsection{General Rigid Tire Models}

The resultant contact forces of the rigid wheel rolling on a compliant terrain are defined by the properties of the soft soil. Hence, the representation of the soft soil is a crucial component in such off-road tire models and the most commonly used soil model is based on Bekker's pressure-sinkage relation (Bekker, 1962). With this Bekker soil model, one can calculate the normal contact pressure that acts along the contact patch, knowing the soil deformation under the wheel.

Due to the soil plasticity and the fact that the tire is rolling forward, the terrain does not bounce back to its original position when losing contact with the wheel. This means that the contact force calculation has to be broken down based on the division of the contact patch into two sections. As can be seen in Figure 3, the rigid wheel compresses the soil up to a maximum sinkage $z_{\max }$ represented by section EF. After going through the compression phase and reaching the maximum compression, the soil passes through the elastic rebound phase in which the elastic 


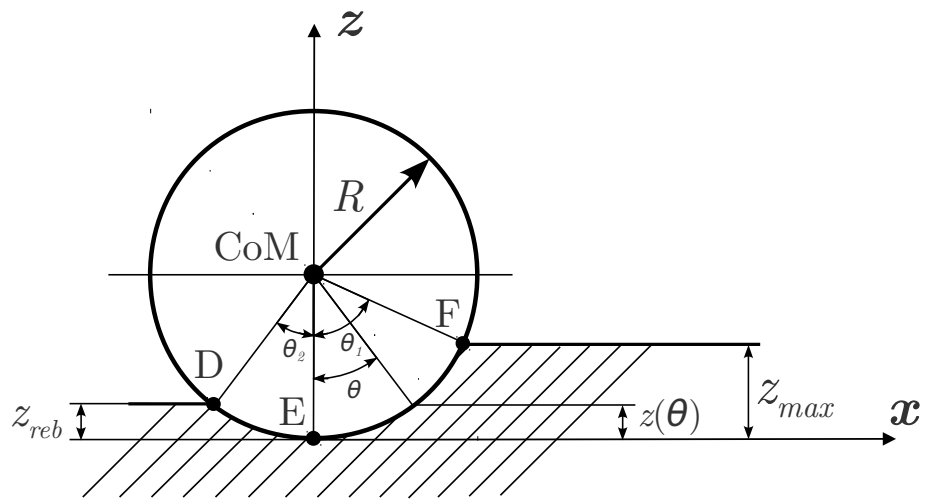

Figure 3 Schematic of rigid wheel model

soil relaxes up to a certain soil rebound $z_{r e b}$ which is represented by the contact patch section DE (Wong, 2010). The contact patch geometry and therefore the contact dynamics are highly dependent on this soil rebound and it can be used to model energy losses due to the plastic behaviour of the soil. These dependencies are studied and presented in the following sections.

With the assumption of a perfect cylinder as tire geometry and a contact geometry as seen in Figure 3, the normal stress distribution $p$ under the wheel can be calculated using the Bekker soil model (Bekker, 1962):

$$
\begin{aligned}
& p(\theta)=\left(\frac{k_{c}}{b}+k_{\phi}\right) z(\theta)^{n} \\
& \text { with } z(\theta)=z_{0}+R(\cos \theta-1)
\end{aligned}
$$

where $k_{c}$ and $k_{\phi}$ are the cohesive and the frictional soil modulus, respectively, and $n$ is the soil deformation exponent. The tire thickness is represented by $b$ and $z$ is the soil deformation over the contact patch where $R$ is the tire radius and $z_{0}$ the maximal soil deformation for the corresponding section of the contact patch.

As in the case of the vertical force, the longitudinal force that defines the tractive effort of a vehicle is limited by the soil mechanics. The tangential forces in the soil and tire interface can be calculated by integrating the shear stress over the contact area. The following exponential model can be used to describe the shear stress-strain curve of soil (Wong, 2003), where $\tau_{\max }$ is calculated using the Mohr-Coulomb failure criteria.

$$
\begin{aligned}
\tau_{x}(\theta)=\tau_{\max } & \left(1-e^{-\frac{j_{x}(\theta)}{K}}\right) \\
\text { with } \tau_{\max } & =c+p(\theta) \tan \phi \\
\text { and } j_{x}(\theta) & =\int_{-\theta_{2}}^{\theta_{1}} R\left[1-\left(1-S_{\text {long }}\right) \cos \theta\right] d \theta
\end{aligned}
$$

Where $j_{x}$ is the shear deformation and $K$ is the shear deformation modulus. The parameters $c$ and $\phi$ represent the soil cohesion and the internal frictional angle, respectively, and $S_{\text {long }}$ is the longitudinal tire slip. The resultant contact 
forces in longitudinal and vertical directions can be calculated by integrating the normal and tangential stress distributions over the contact patch. Since both of these stress distributions possess parts in both longitudinal and vertical directions, these parts have to be taken into account and added up accordingly for each of the tire forces. The results are the longitudinal and vertical tire forces which can be calculated as shown in Eqn 9 and 10 respectively.

$$
\begin{aligned}
& F_{x}=\int \tau_{x}(\theta) \cos \theta d \theta-\int p(\theta) \sin \theta d \theta \\
& F_{z}=\int p(\theta) \cos \theta d \theta+\int \tau_{x}(\theta) \sin \theta d \theta
\end{aligned}
$$

To generate closed-form expressions for these integrals, the volumetric contact model can be used for normal force calculation as explained in the following section.

\section{Volumetric Tire Model Approaches}

Assuming a perfectly linear-elastic soil that fully rebounds back to its original position, the Bekker model resembles an elastic foundation with linear-elastic spring properties. Therefore, the volumetric model is used to replace the Bekker soil model to find a closed form solution for the normal contact force. The advantage is that if the geometry of each colliding body is known, the volume can be easily calculated. Furthermore, it would already carry the information of the shapes in contact with each other, which can not be captured by a depth of penetration model, and would have to be integrated by the Bekker model which takes time. Replacing the normal force integrals from the Bekker model with closed form expressions of the volumetric model can significantly reduce computational time of vehicle dynamics simulations.

As previously mentioned, the volumetric model equals the Bekker model assuming that the elastic foundation stiffness is equal to the total Bekker stiffness and the Bekker exponent $m=1$. However, the soil does not behave the same way in rebound as it does when being compacted. Therefore, a number of different soil models can be suggested according to Wong's method (Wong, 2003), in which the tire contact patch is separated into front and rear sections and the soil is compacted up to a maximum compression and relaxed up to a certain rebound, respectively. In this article, three different tire models with various volumetric contact models are tested with respect to the soil plasticity:

1. Tire Model I: different soil properties in compression and relaxation mode

2. Tire Model II: same soil properties in compression and relaxation mode

3. Tire Model III: gradually changing soil properties

All of these tire models are based on a perfect cylinder in contact with a smooth and soft terrain. This assumption is chosen for simplicity of the preliminary results, but is not a restriction to the volumetric contact model. As previously stated, the volumetric model is used to replace the normal contact force. The tangential force is still calculated by integrating the shear stress over the contact patch. The 
normal force can be calculated with Eqn 4 as presented earlier and the contact normal vector is determined using Eqn 11.

$$
\begin{aligned}
& \mathbf{n}=\sin \varphi \hat{\imath}+\cos \varphi \hat{k} \\
& \text { with } \varphi=\arctan \frac{\mathbf{r}_{c, x}}{\mathbf{r}_{c, z}}
\end{aligned}
$$

where $\varphi$ is the angle between the normal vector and the vertical direction and $\mathbf{r}_{c}$ is the position vector of the penetration volume centroid with respect to the wheel hub frame. The normal force component in $x$-direction is the soil resistance due to compaction and the component of the normal force in $z$-direction represents the vertical tire force which supports the wheel load given by the weight and the dynamics of the rover. To determine the tire forces, the components of the normal force have to summed up with the components of the tangential force, which is still calculated by integrating the shear stress distribution as explained earlier. The $x$-component of the tangential force represents the traction force generated in the contact patch and the tangential force component in $z$-direction also supports the load of the wheel. Finally, the complete tire forces in longitudinal and vertical directions are calculated using Eqn 12 and Eqn 13 respectively.

$$
\begin{aligned}
& F_{x}=F_{\text {trac }}-R_{c}=\text { Drawbar Pull } \\
& \quad \text { with } F_{\text {trac }}=\int \tau_{x}(\theta) \cos \theta d \theta \text { and } R_{c}=k_{v} V \sin \varphi \\
& F_{z}=k_{v} V \cos \varphi+\int \tau_{x}(\theta) \sin \theta d \theta
\end{aligned}
$$

where $F_{\text {trac }}$ is the generated traction force and $R_{c}$ is the soil compaction resistance. This method can be used for all three tire models. However, the variations in the described volumetric rigid wheel representations are modelled using different contact geometries for each of the tire models. The difference is simply the shape of the interpenetration volume and its centroid which together define the absolute value and direction of the normal contact force. The soil plasticity is caused by the compaction due to the rover wheel rolling on the soft soil. The amount of plastic deformation left behind the tire is represented by the soil rebound whose impact on the traction forces as well as the soil resistance is evaluated in the following section using different rigid volumetric wheel models. It should be noted that the presented models do not predict the plastic deformation of the soil, but the soil rebound is assumed to be given and varied to evaluate its impact on the tire dynamics. Also, the three different tire models are explained in detail in the following sections. The models are also compared against experimental data taken from Scharringhausen et al. (2009), which is based on a single wheel testbed. This testbed is located at DLR facilities in Bremen, Germany and consists of a $3 m$ long soil bin and a sled that drives the rover wheel through the soil. A rigid smooth wheel (radius $R=125 \mathrm{~mm}$, width $b=100 \mathrm{~mm}$ ) with a vertical wheel load of $70 \mathrm{~N}$ was translated at a velocity of $v_{x}=10 \mathrm{~mm} / \mathrm{s}$ through fine and dry quartz sand. Numerous measurements were taken by prescribing the angular velocity of the tested wheel in order to create wheel slip varying from $0 \%$ to $95 \%$. For the experiment, the longitudinal slip was defined as shown in Eqn 14.

$$
s_{\text {long }}=1-\frac{v_{x}}{R \omega_{y}}
$$


where $v_{x}$ is the longitudinal velocity and $\omega_{y}$ is the wheel spin. The same slip definition is used in the calculation of the tangential forces of the presented tire models.

\subsection{Tire Model I}

The first version of a rigid rover wheel volumetric model is based on the fact that the soil properties differ when the soil is in relaxation mode compared to a soil under compression. A schematic of the resulting penetration volume is illustrated in Figure 4.

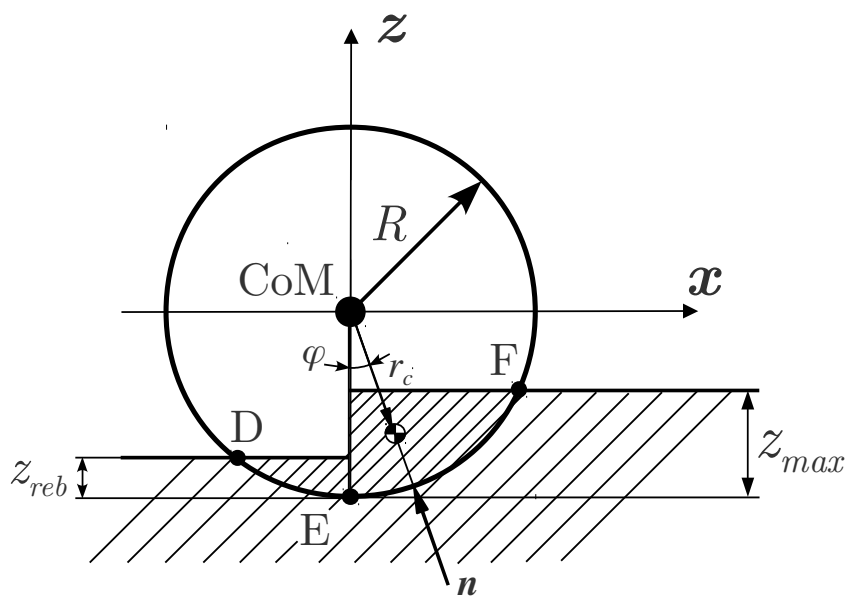

Figure 4 Schematic of tire model I

The schematic shows that the soil is compressed up until the maximum sinkage $z_{\max }$ (shown in the front section of the tire) from which it rebounds up to a certain soil rebound $z_{r e b}$ (shown in the rear section of the tire). This means that the normal force under the rear section is based on pressure distribution where the maximal soil displacement $z_{0}$ is equal to the soil rebound $z_{r e b}$. The volumetric normal tire force is calculated using the closed-form expression in Eqns 4 and 11. The difference between the model and the traditional Bekker model can be seen in the following equation. To obtain the same results as in the volumetric tire model, the normal force would have to be determined by integrating the Bekker pressuresinkage relation as follows:

$$
\begin{aligned}
& \mathbf{F}_{n, \text { Bekker }}= {\left[k_{\text {Bekker }}\left(-\int_{0}^{\theta_{1}} z_{1}(\theta) \sin \theta d \theta+\int_{0}^{\theta_{2}} z_{2}(\theta) \sin \theta d \theta\right)\right] \hat{\imath} } \\
&+ {\left[k_{\text {Bekker }}\left(\int_{0}^{\theta_{1}} z_{1}(\theta) \cos \theta d \theta+\int_{0}^{\theta_{2}} z_{2}(\theta) \cos \theta d \theta\right)\right] \hat{k} } \\
& \text { with } z_{1}(\theta)=z_{\text {max }}+R(\cos \theta-1) \text { and } z_{2}(\theta)=z_{\text {reb }}+R(\cos \theta-1)
\end{aligned}
$$

where $\theta_{1}$ and $\theta_{2}$ are the contact patch angles of the front and rear sections of the tire respectively and are illustrated in Figure 3. To test the volumetric rigid 
tire model for different soils, the resultant drawbar pull $F_{x}$ for this tire model is evaluated for various amounts of soil rebound using Eqns 12 and the penetration volume indicated in the schematic of Figure 4. The drawbar pull is the effective driving force that is generated in the contact patch. It is calculated by summing up the traction and resistance forces, as shown in Eqn 12. For the measured tire states, the soil compaction resistance $R_{c}$ is the dominant dissipative force. Therefore, it is the only resistive force considered in tire models presented in this paper. The results are plotted and compared against experimental data retrieved from (Scharringhausen et al., 2009) and can be seen in Figure 5.

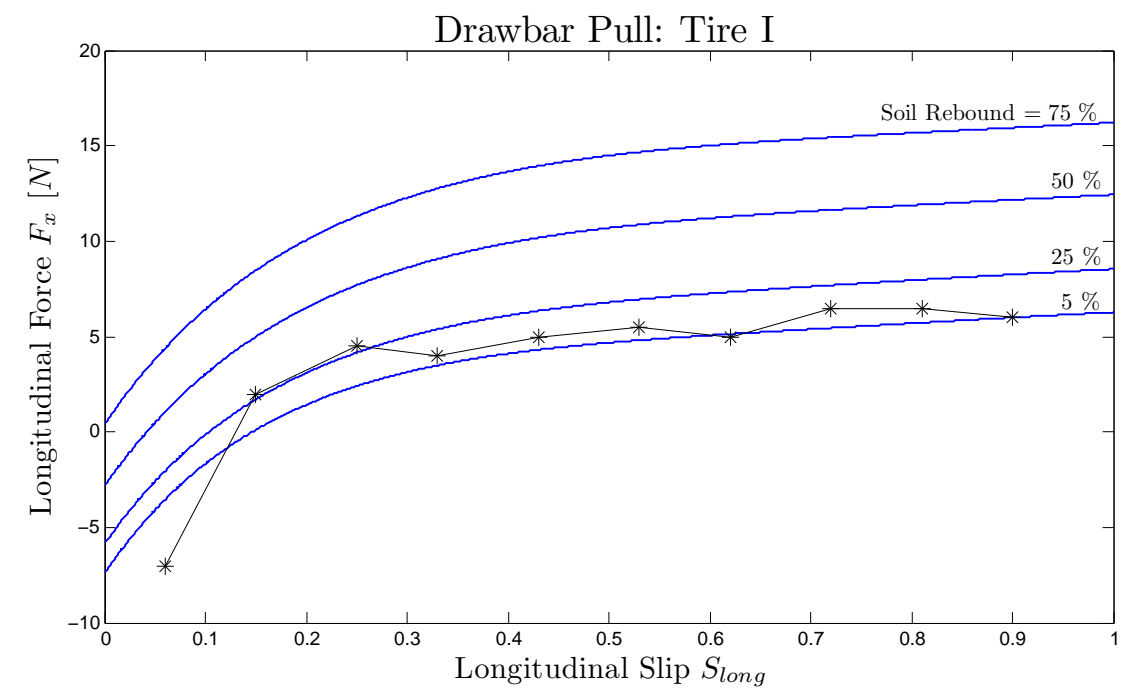

Figure 5 Tire I: Drawbar Pull vs. Slip with respect to various soil rebounds in comparison with a tested rigid tire

The continuous lines illustrate the drawbar pull as predicted by this tire model and the measurements are noted by the asterisks. In the results plot, shown in Figure 5, can be seen that the soil rebound significantly impacts the effective traction force generated in the contact patch. Also, the tire model considering a rather low amount of soil rebound is in best agreement with the experimentally gained results, as it was expected due to the naturally low rebound properties of the dry quartz sand.

\subsection{Tire Model II}

This version of the volumetric rigid tire model is based on the assumption that the soil possesses the same properties along the full extent of the contact patch. A schematic of this tire model II can be seen in Figure 6.

It can be seen that the soil is compressed all the way from the original soil position throughout the entire contact patch even after it passes through the maximum sinkage $z_{\max }$. The soil rebound then influences the normal contact force in a way that the remaining penetration volume is cut off when the tire loses 


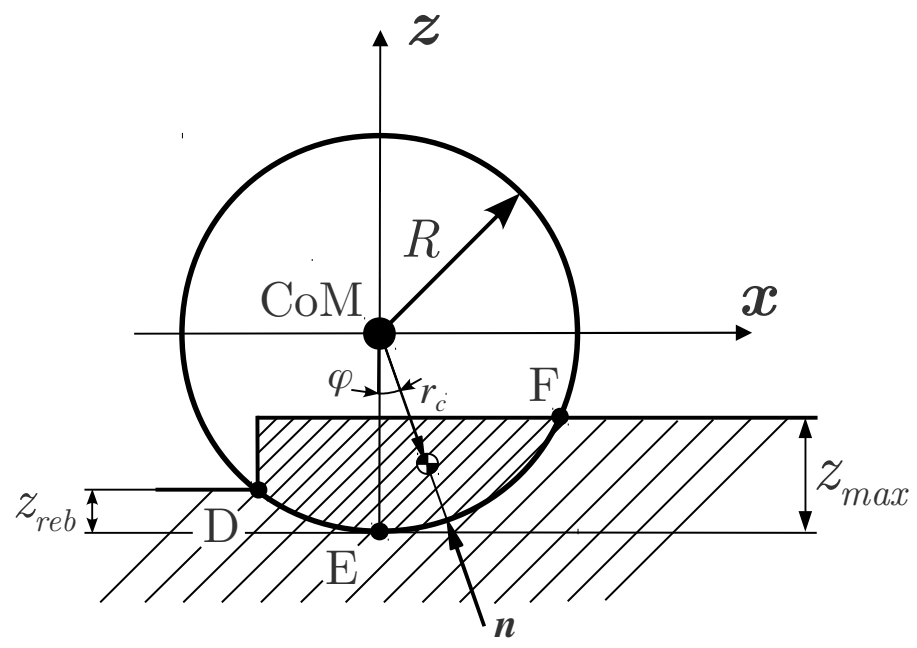

Figure 6 Schematic of tire model II

contact with the soil. Calculating the normal force with the volumetric closedform expression considering the contact geometry as shown in Figure 6 would be equivalent to a normal force determined by integrating the Bekker pressure-sinkage relation as follows:

$$
\begin{gathered}
\mathbf{F}_{n, \text { Bekker }}=\left[k_{\text {Bekker }} \int_{-\theta_{2}}^{\theta_{1}} z(\theta) \sin \theta d \theta\right] \hat{\imath}+\left[k_{\text {Bekker }} \int_{-\theta_{2}}^{\theta_{1}} z(\theta) \cos \theta d \theta\right] \hat{k} \\
\text { with } z(\theta)=z_{\max }+R(\cos \theta-1)
\end{gathered}
$$

To evaluate the influence of the soil rebound of this type of volumetric tire model, the resultant drawbar pull is again calculated with Eqn 12 but this time considering the penetration volume indicated in the schematic of Figure 6 . The different results for various amounts of soil rebound are plotted and also compared against the previously indicated experiments (see Figure 7).

The plot of results seen in Figure 7 again shows a significant impact of the soil rebound on the effective traction force that can be generated in the contact patch. Contrary to tire model I, the impact of the soil rebound in this tire model appears to be more significant in the lower soil rebound region. This is due to the fact that the penetration volume barely changes for small variations in soil rebound for higher amounts of rebound. Also, the results of this tire model show that the agreement with the experimental results is not as good as in the previous version of the volumetric tire model. This suggests that treating the soil as a material with unchanged properties over the entire contact patch may not lead to realistic results.

\subsection{Tire Model III}

In this particular implementation of the volumetric rigid wheel model, the assumption of a gradually decreasing compression of the soil under the wheel is made. A schematic of this tire model III is illustrated in Figure 8. 


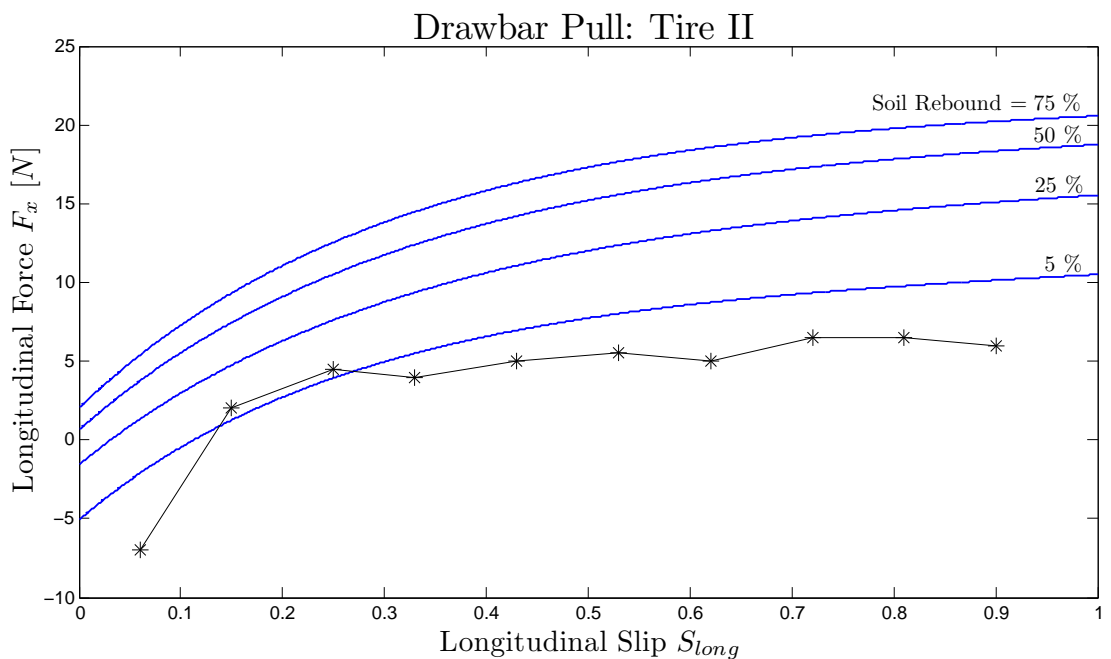

Figure 7 Tire II: Drawbar Pull vs. Slip with respect to various soil rebounds in comparison with a tested rigid tire

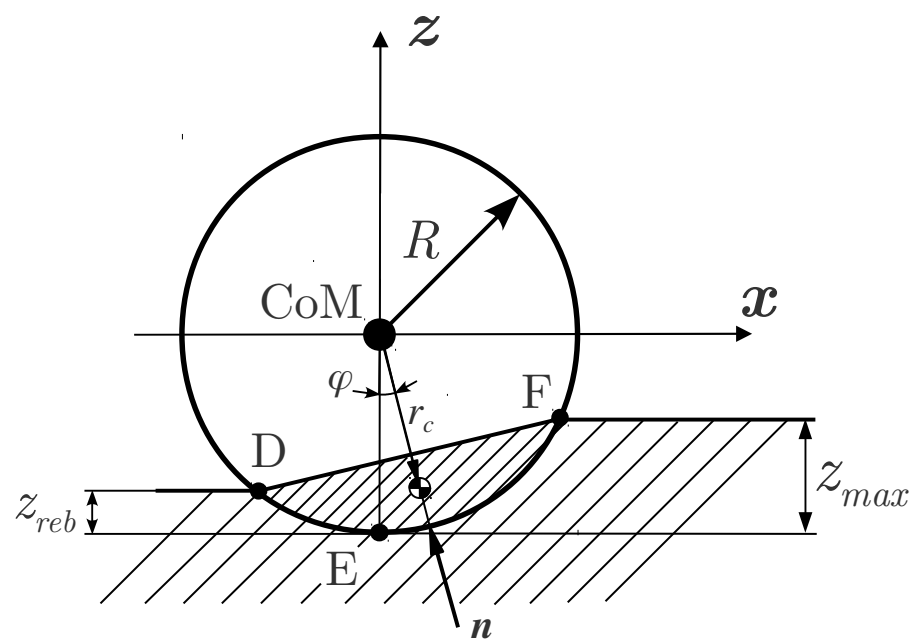

Figure 8 Schematic of tire model III 
The schematic shows the penetration volume used for this version of the tire model which is defined by a line connecting points $\mathrm{D}$ and $\mathrm{F}$. This line is denoted $g(\theta)$. The soil rebound then directly influences the normal force calculation and its direction since the contact normal is directly related to the slope of this line. This is equivalent to a normal force determined by integrating the Bekker pressuresinkage relation as follows:

$$
\begin{aligned}
& \mathbf{F}_{n, \text { Bekker }}=\left[k_{\text {Bekker }} \int_{-\theta_{2}}^{\theta_{1}}(z(\theta)-g(\theta)) \sin \theta d \theta\right] \hat{\imath} \\
&+ {\left[k_{\text {Bekker }} \int_{-\theta_{2}}^{\theta_{1}}(z(\theta)-g(\theta)) \cos \theta d \theta\right] \hat{k} } \\
& \text { with } z(\theta)=z_{\text {max }}+R(\cos \theta-1)
\end{aligned}
$$

Again, Eqns 12 and 13 are used to calculate the tire forces based on this particular penetration volume. The results are plotted and compared as in the previous volumetric tire models which can be seen in Figure 9.

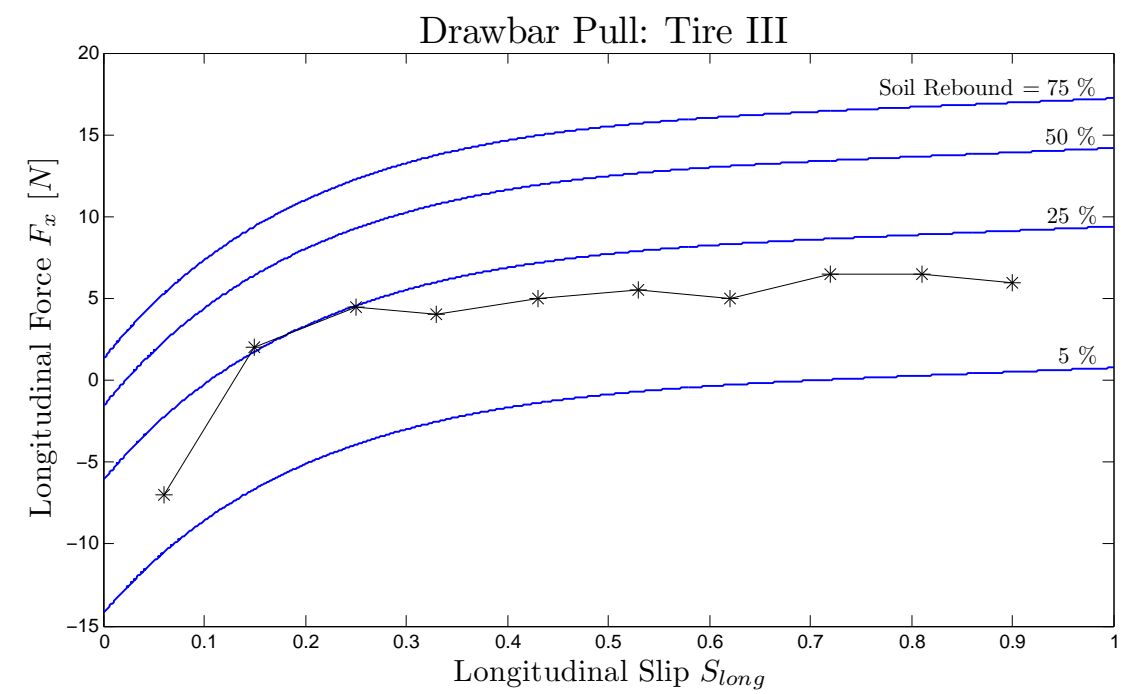

Figure 9 Tire III: Drawbar Pull vs. Slip with respect to various soil rebounds in comparison with a tested rigid tire

As in the earlier displayed volumetric tire models, the results of this 3rd version reveal a similar significance of the soil rebound. However, it can be seen that the effective longitudinal force at lower values of soil rebound is significantly lower than in the previous tire models. This suggests that this tire model predicts higher resistance forces than the previous tire models predicted for soil with little rebound. The impact of the relaxation properties of the soil on each of the components of the longitudinal force and the drawbar pull itself are discussed in more detail in the following section. 


\section{Discussion}

Besides the direct influence of the soil rebound on the normal force calculation in all three of the discussed volumetric rigid tire models, the amount of soil rebound also indirectly impacts the calculation of the tangential forces. Therefore, both the soil compaction resistance $R_{c}$ and the traction force $F_{t r a c}$, which are the two force components of the effective longitudinal force $F_{x}$, are influenced by the plastic deformation of the soil that remains after the wheel rolls over the soft terrain.

The soil rebound directly influences the normal force calculation by defining the penetration volume and shifting the centroid. Assuming the same wheel load, the soil rebound influences the normal force by changing the direction of the contact normal. The angle between the contact normal and the vertical direction decreases with increasing soil rebound. A smaller angle from the vertical direction means that a larger amount of the normal force supports the wheel load and a smaller soil compaction resistance is the result. Another result of this scenario is the decreasing tire sinkage and thus a smaller contact patch and generated traction force. This tendency is counteracted by the assumption that an increase of soil rebound also increases the contact patch size as a results of the altered contact patch angles $\theta_{1}$ and $\theta_{2}$ (see Figure 3). To evaluate the compaction resistance and the traction force, these forces are calculated for each of the volumetric tire models at a constant slippage of $25 \%$ and varying the soil rebound from $0 \%$ to $100 \%$ of the maximum sinkage. The wheel load is kept at a constant value of $70 \mathrm{~N}$ and the results can be seen in Figures 10 and 11. Finally, the effective driving force $F_{x}$, also known as the drawbar pull, is calculated and evaluated under the same conditions. The results can be seen in the plot of Figure 12.

Figure 10 shows the effect on soil compaction resistance when the soil rebound is increased from $0 \%$ to $100 \%$ for each tire model. As expected from the geometry

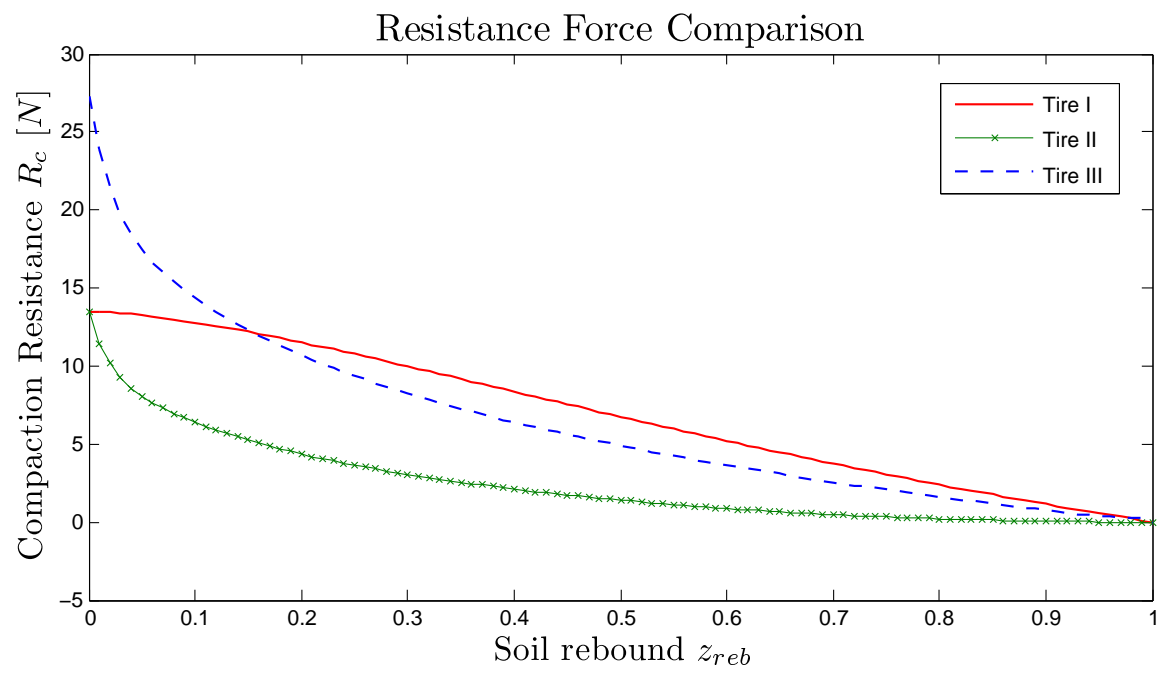

Figure 10 Comparison of compaction resistant with respect to soil rebound

of the penetration volume, the first two tire models start off from the same 
resistance force for zero soil rebound whereas tire model III predicts a significantly larger resistance for soil with no rebound. For all three versions of the tire model, the plots depict a decreasing trend with respect to an increasing soil rebound until the soil resistance settles to zero for $100 \%$ of soil rebound. This is due to the fact that the contact normals in each tire model are aligned with the vertical direction for this soil state which is a result of the geometry of the penetration volume. Whereas tire I almost linearly decreases with increasing soil rebound, the plots show that tire model II and III are more sensitive to changes in the lower soil rebound region as suggested by the results shown in the previous section of this report.

Figure 11 shows the effect on the generated traction force when the soil rebound is increased from $0 \%$ to $100 \%$. It can be seen that the traction force

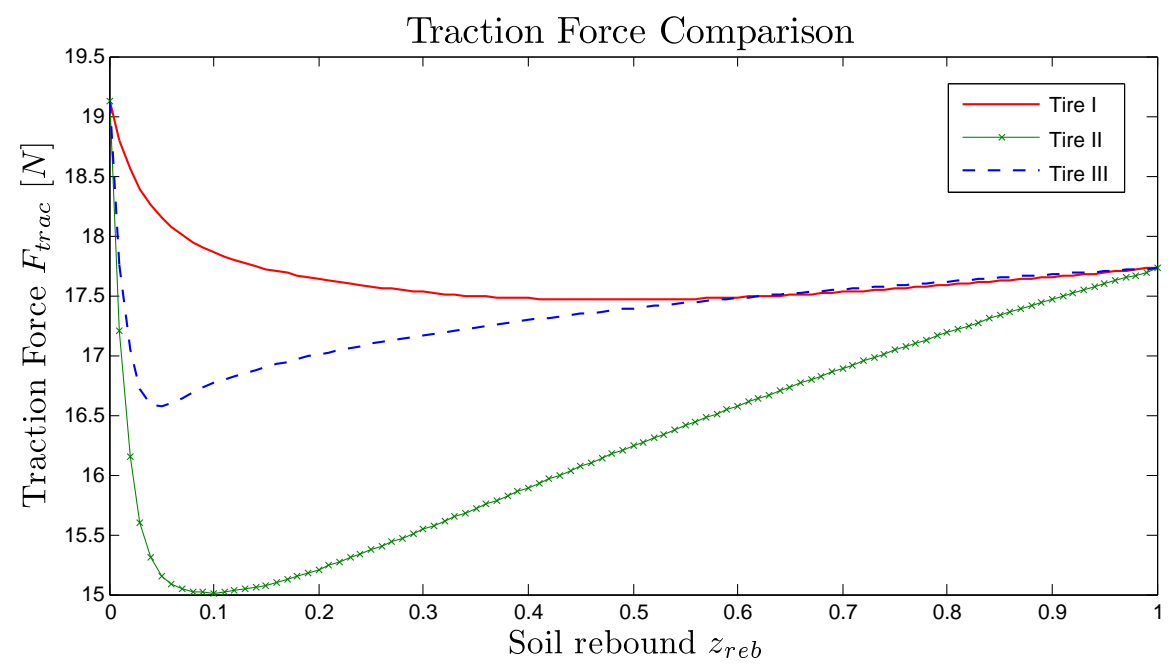

Figure 11 Comparison of traction force with respect to soil rebound

for all three tire models begins with the same force value and decreases rapidly afterwards. This sudden drop of traction force is due to the decreasing size of the contact patch due to the increase in soil rebound discussed earlier. This effect appears to have the biggest impact on tire model II. Also, it seems to have less but still quite substantial impact on tire model III. The first tire model shows the least sensitivity to this effect. This is also due to the fact that the three tire models show a reversed sensitivity to the counteracting increase of the contact patch size as a result of the changing penetration volume geometry. Eventually this counteracting effect overtakes the decrease in contact patch size and all three tire models settle to the same amount of traction force at a value of $100 \%$ of soil rebound.

As a conclusive diagram, the drawbar pull is plotted against the soil rebound which can be seen Figure 12. The plot of the drawbar pull shows that the volumetric rigid wheel models I and II predict similar effective longitudinal forces for soil with very low and very high relaxation properties, but diverge significantly for other soils. The predicted drawbar pull in the 3rd version of the volumetric rigid tire model appears to be significantly lower for soils with low rebound 
properties. For soils with soil rebound larger than $25 \%$, the effective driving force in tire model III appears to form an average of the two previous tire models.

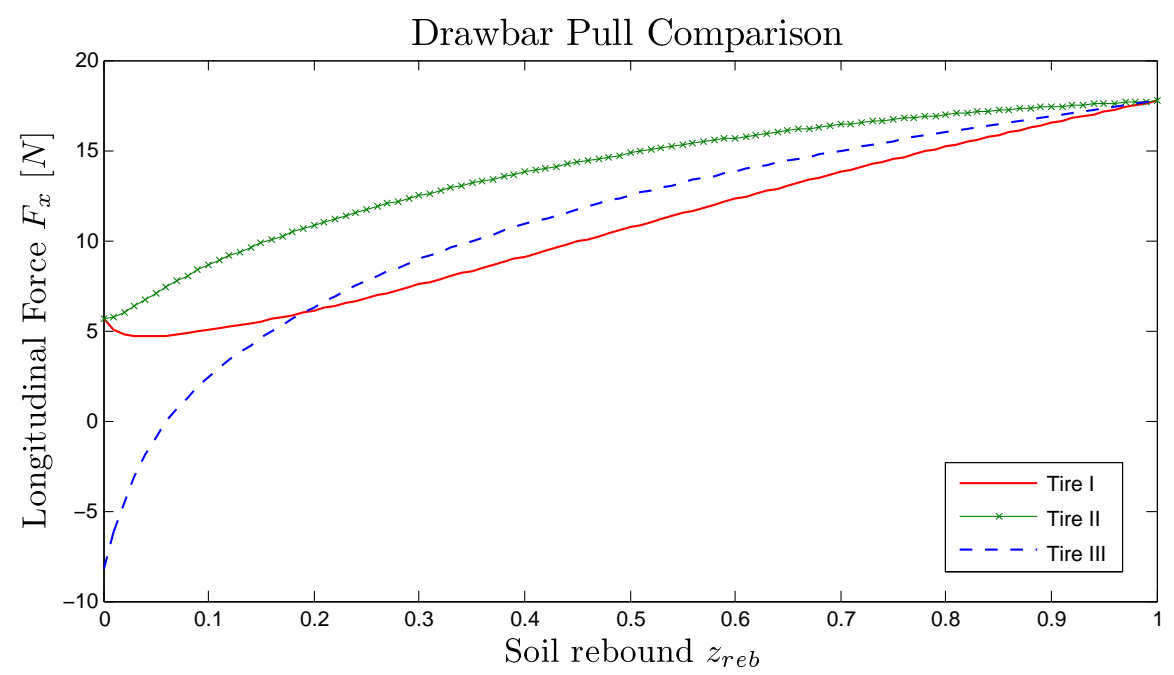

Figure 12 Comparison of drawbar pull with respect to soil rebound

\section{Conclusions}

In conclusion, three different volumetric rigid tire models are created. For these tire models, the normal force calculation is based on the penetration volume and its centroid location. The advantage of a volume-based calculation of the normal force is that a closed-form expression can be found which replaces the computationally time-consuming integrals of Bekker's pressure-sinkage relation. The difference between the three developed rigid tire models is the geometry of the penetration volume. The different contact geometries are chosen to model differences in the way the plastic deformation of the soil influences the tire dynamics. The quantity used to represent this plastic deformation is the amount of soil rebound at which the tire loses contact with the ground. All three volumetric models are simulated and tested with respect to this soil rebound. Also, the results of each tire are compared against experimental results obtained from a single wheel testbed. It is shown that the modelled rigid wheel results are confirmed by the experimental results which also confirms the hypothesis that soils under compression behave differently than soils in rebound phase. Furthermore, it is shown that the soil rebound influences the resistive force and traction force and, as a result, the effective driving force that can be generated in the contact patch.

For future work, it should be noted that this article presents tire models for a rover wheel in contact with smooth ground which are used as preliminary results in the development for tire models in contact with 3-dimensional terrains. Furthermore, it is shown that the normal force calculation can be replaced by a volumetric contact model approach and the tangential contact force model will also 
have to be replaced to achieve the full simulation time advantage of a volumetric rover wheel model. Also, the linear elastic foundation model can be replaced by a hyperelastic foundation and the resultant hypervolumes can be used in a volumetric tire model in contact with nonlinear soils.

\section{Nomenclature}

\begin{tabular}{|c|c|c|}
\hline Symbol & Description & Unit \\
\hline$\phi$ & Internal friction angle & rad \\
\hline$\varphi$ & Normal direction angle & rad \\
\hline$\theta$ & Contact patch angle & rad \\
\hline$\rho_{s}$ & Position of point on the contact surface & $m$ \\
\hline$\tau_{\max }$ & Maximal shear stress & $M P a$ \\
\hline$\tau_{R}$ & Rolling resistance moment & $N m$ \\
\hline$\omega$ & Angular velocity vector & $\mathrm{rad} / \mathrm{s}$ \\
\hline$\tau_{x}$ & Shear stress in $x$-direction & $M P a$ \\
\hline$\omega_{y}$ & Wheel spin & $\mathrm{rad} / \mathrm{s}$ \\
\hline$b$ & Tire width & $m$ \\
\hline$d$ & Damping factor & $N s / m$ \\
\hline$f_{S}$ & Elastic foundation deformation depth & $m$ \\
\hline$F_{n}$ & Normal contact force & $N$ \\
\hline$F_{t r a c}$ & Traction force & $N$ \\
\hline$F_{x}$ & Longitudinal tire force & $N$ \\
\hline$F_{z}$ & Vertical tire force & $N$ \\
\hline$h_{f}$ & Depth of Winkler elastic foundation & $m$ \\
\hline$j_{x}$ & Longitudinal shear displacement & $m$ \\
\hline $\mathbf{J}_{s}$ & Inertia tensor of penetration volume & $m^{5}$ \\
\hline$k_{\phi}$ & Frictional shear modulus & $N / m^{n+2}$ \\
\hline$k_{\text {Bekker }}$ & Bekker soil stiffness & $N / m^{n}$ \\
\hline$k_{c}$ & Cohesive shear modulus & $N / m^{n+1}$ \\
\hline$k_{f}$ & Winkler elastic foundation modulus & $N / m$ \\
\hline$k_{v}$ & Volumetric stiffness & $N / m^{3}$ \\
\hline$K$ & Shear deformation modulus & $m$ \\
\hline$n$ & Bekker soil deformation coefficient & - \\
\hline $\mathbf{n}$ & Normal direction vector & - \\
\hline$p$ & Bekker soil pressure & $M P a$ \\
\hline $\mathbf{r}_{c}$ & Centroid location vector & $m$ \\
\hline$r$ & Loaded tire radius & $m$ \\
\hline$R$ & Wheel radius & $m$ \\
\hline$R_{c}$ & Resistance due to compaction of the soil & $N$ \\
\hline$S$ & Contact surface & - \\
\hline$S_{\text {long }}$ & Longitudinal slip & - \\
\hline$v_{n}$ & Normal relative velocity of contact bodies & $\mathrm{m} / \mathrm{s}$ \\
\hline$v_{x}$ & Tire velocity in $x$-direction & $\mathrm{m} / \mathrm{s}$ \\
\hline$V$ & Penetration volume & $m^{3}$ \\
\hline$z$ & Soil compression & $m$ \\
\hline$z_{0}$ & Nominal soil compression & $m$ \\
\hline$z_{\max }$ & Maximal soil compression & $m$ \\
\hline$z_{r e b}$ & Soil rebound & $m$ \\
\hline
\end{tabular}




\section{References}

Bauer, R., Leung, W. and Barfoot, T. (2005) 'Experimental and Simulation Results of Wheel-Soil Interaction for Planetary Rovers', International Conference on Intelligent Robots and Systems (IEEE/RSJ — IROS 2005), Edmonton, Canada, 2005.

Bekker, M.G. (1962) 'Theory of Land Locomotion: The mechanics of vehicle mobility', University of Michigan Press, 1962.

Bekker, M.G. (1969) 'Introduction to Terrain-Vehicle Systems', University of Michigan Press, 1969.

Boos, M. and McPhee, J. (2010) 'Volumetric Contact Models and Experimental Validation', International Conference on Multibody System Dynamics (IMSD 2010), Lappeenranta, Finland, 2010.

Gonthier, Y. (2003) 'On the implementation of Coulomb friction in a contact model based on volumetric properties', 6th International Conference on Multibody Systems, Nonlinear Dynamics and Control (ASME/IDETC 2003), Las Vegas, USA, 2003.

Gonthier, Y. (2007) 'Contact Dynamics Modelling for Robotic Task Simulation', PhD Thesis, University of Waterloo, Canada, 2007.

Harnisch, C., Lach, B., Jakobs, R., Troulis, M. and Nehls, O. (2005) 'A new tyresoil interaction model for vehicle simulation on deformable ground', Vehicle System Dynamics, Vol. 43, pp.384-394.

Janosi, Z. and Hanamoto, B. (1961) 'Analytical Determination of Drawbar Pull as a Function of Slip for Tracked Vehicles in Deformable Soils', 1st International Conference on Terrain-Vehicle Systems (ISTVS 1961), Torino, Italy, 1961.

Johnson, K. (1987) 'Contact Mechanics', Cambridge University Press, 1987.

Onafeko, O. and Reece, A.R. (1967) 'Soil Stresses and Deformations beneath Rigid Wheels', Journal of Terramechanics, Vol. 4, No. 1, pp.59-80.

Scharringhausen, M., Beermann, D., Krömer, O. and Richter, L. (2009) 'A Wheel-Soil Interaction Model for Planetary Application', 11th European Regional Conference on Terrain-Vehicle Systems (ISTVS 2009), Bremen, Germany.

Scharringhausen, M., and Beermann, D., Krömer, O. and Richter, L. (2009) 'Single Wheel Tests for Planetary Applications at DLR Bremen', 11th European Regional Conference on Terrain-Vehicle Systems (ISTVS 2009), Bremen, Germany.

Wong, J.Y. (1967) 'Behaviour of soil beneath rigid wheels', J. of Agric. Engng. Res., Vol. 12, No. 4, pp.257-269.

Wong, J.Y. and Reece, A.R. (1967) 'Prediction of Rigid Wheel Performance Based on the Analysis of Soil-Wheel Stresses Part I. Performance of Driven Rigid Wheels', Journal of Terramechanics, Vol. 4, No. 1, pp.81-98.

Wong, J.Y. and Reece, A.R. (1967) 'Prediction of Rigid Wheel Performance Based on the Analysis of Soil-Wheel Stresses Part II. Performance of Towed Rigid Wheels', Journal of Terramechanics, Vol. 4, No. 2, pp.7-25.

Wong, J.Y. (2003) 'Theory of ground Vehicles', J. Wiley 3rd Edition, 2003.

Wong, J.Y. (2010) 'Terramechanics and Off-Road Vehicle Engineering - Terrain Behaviour, Off-Road Vehicle Performance and Design', Elsevier Ltd. 2nd Edition, 2010 .

Yong, R.N. and Fattah, E.A. (1975) 'Influence of Contact Characteristics on Energy Transfer and Wheel Performance on Soft Soil', 5th International Conference on Terrain-Vehicle Systems (ISTVS 1975), Detroit, USA, 1975. 